\title{
Effect of Sheep Nano Antibody on Muscle Movement of Human Tissue Flap
}

Y. F. YU*

Institute of Physical Education, Tonghua Normal University, Tonghua, Jilin 134002, China

Yu et al.: Effect of Sheep Nano Antibody on Muscle Movements

In this study, the effect of sheep nano antibody on the movement of human tissue flap muscle is studied. Firstly, the non-toxic effect of bivalent sheep nano antibody on mouse muscle cells is investigated and then the effect of the antibody on the movement of mouse tissue flap muscle is analyzed. In the repeated experiment of injecting bivalent sheep myostatin nano antibody into mice, 30 healthy Kunming mice of 1 mo old and weighing $15 \mathrm{~g}$ without specific pathogen are selected. When using 3-(4,5-dimethylthiazol2-yl)-2,5-diphenyl tetrazolium bromide method to detect the myoblast toxicity of mouse immortalized mouse myoblast cell line $\mathrm{C2C12}$, phosphate buffered saline control group, blank control group and the observation group with concentration of $10,20,30$ and $60 \mu \mathrm{g} / \mathrm{ml}$ of bivalent nano antibody membrane proteome array are set respectively. In the repeated experiment of bivalent sheep myostatin nano antibody on mice, it is divided into phosphate buffered saline control group, monovalent group and bivalent group. Each animal in phosphate buffered saline group is injected with $30 \mu \mathrm{l}$ phosphate buffered saline, each animal in monovalent group is injected with $30 \mu \mathrm{l}(60 \mu \mathrm{g})$ monovalent sheep myostatin nano antibody and each animal in bivalent group is injected with $30 \mu \mathrm{l}(60 \mu \mathrm{g})$ bivalent sheep myostatin nano antibody. The weight of the single hind limb of the bivalent sheep myostatin nano antibody group is higher than that of the monovalent sheep myostatin nano antibody group and the control group, and the muscle morphology of the hind limb of the bivalent sheep myostatin nano antibody group is significantly higher than that of the monovalent sheep myostatin nano antibody group and the control group, which is more plump. The results show that sheep nano antibody is non-toxic to human tissue flap muscle, which can promote the growth of human tissue flap muscle and enhance muscle movement ability.

Key words: Sheep nano antibody, tissue flap, cytotoxicity, muscle morphology, myostatin

With the continuous exploration of biological science research, the biomedical tool based on antibody is put forward ${ }^{[1,2]}$ and continuous development and research on it are made. It is concluded that the gene coding of nano antibody is highly similar to that of human and has weak immunogenicity in human body, so it is widely used in medical research ${ }^{[3-6]}$. The movement of human tissue flap muscle studied in this paper is to enhance the shape of muscle through nano antibody, so as to affect the movement ability of muscle and provide powerful support for the phenomenon of human muscle atrophy in current treatment medicine ${ }^{[7]}$. Muscle atrophy refers to the decrease of skeletal muscle volume due to the decrease of muscle fiber number or volume. It is a disease of progressive muscle weakening and atrophy, common in arms and thighs. Muscular atrophy patients lie in bed for a long time due to muscular atrophy and muscle weakness, which reduces the quality of life of patients and poses a great threat to their lives ${ }^{[8-10]}$.

Since the advent of antibody technology, people think that the potential of antibody in the treatment of diseases is infinite. Conventional drugs will have side effects or toxic reactions when they are used in the treatment of certain diseases and the substance produced by the organism is the antibody itself, which does not need to worry too much about the problem of toxicity, so it is widely used in medicine. However, the traditional single antibody has the problems of large volume, complex preparation process and poor stability ${ }^{[11]}$, which makes it inconvenient to use. Nano antibody belongs to one kind of antibody, which can bind antigen well. Compared with the traditional single antibody, the ability of binding with antigen has not been reduced

*Address for correspondence

E-mail: thsyyyf615@126.com 
and it can replace the traditional single antibody to bind with antigen. In addition, nano antibody also avoids the shortcomings of the traditional single antibody. Nano antibody has small amount of components, high water solubility and stability, and is easier to use in medicine. In addition, Myostatin (MSTN) is widely studied in medicine and zoology. MSTN can promote the development and growth of animal skeletal muscle, improve the atrophy of animal muscle and promote the growth and development of animal muscle. Therefore, this paper studies the bivalent sheep MSTN nano antibody, which combines MSTN and nano antibody technology. Through experiments, it is verified that the bivalent sheep MSTN nano antibody is safe, non-toxic and has a high effect on promoting muscle growth, providing an effective basis for the treatment of muscle atrophy symptoms ${ }^{[12,13]}$.

There are many pathological muscle atrophy phenomena in medicine, for example, after amputation of lower limbs, the victory environment and mechanical environment of muscle tissue have changed significantly, the growth of muscle and the adaptability of the stump have been greatly affected, long-term development will lead to the phenomenon of muscle atrophy of the stump, which has a great impact on muscle strength, sports and daily life ability. Therefore, how to slow down muscle atrophy and restore exercise ability has become a very concerned problem in the field of medical rehabilitation.

In order to save the life of patients with muscle atrophy and improve their quality of life, bivalent sheep nano antibody is used in this paper, which affects the shape and weight of limb muscles, so as to gradually restore the muscle shape of patients with muscle atrophy and improve their muscle movement ability. Moreover, through the experiment, bivalent sheep nano antibody has high safety and low side effects after long-term use $^{[11]}$, so it can be used in medicine to treat muscular atrophy symptoms and improve the movement ability of muscles.

\section{MATERIALS AND METHODS}

\section{Materials:}

Experimental animals: 30 Kunming mice are bought from Xinjiang Medical University. These mice are 1 mo old, with a weight of $15 \mathrm{~g}$, healthy without specific pathogens.

Reagents: Main reagents used in this study are given in Table 1.

Instruments: Main instruments used in this study are given in Table 2.

\section{TABLE 1: MAIN REAGENTS}

\begin{tabular}{|c|c|}
\hline Reagents & Source \\
\hline $\begin{array}{l}\text { Concentrated 3,3'-Diaminobenzidine (DAB) color developing } \\
\text { kit }\end{array}$ & $\begin{array}{l}\text { Purchased from Beijing Zhongshan Jinqiao Biotechnology } \\
\text { co., Ltd }\end{array}$ \\
\hline DMEM cell culture medium & Purchased from Gibco \\
\hline Fetal Bovine Serum (FBS) & Purchased from Gibco \\
\hline Cell culture plate & Purchased from NUNC Denmark \\
\hline Protein molecular weight Marker & From $\mathrm{MBI}$ Fermentas \\
\hline Rabbit anti-MSTN polyclonal antibody & Purchased from Abcam \\
\hline $\begin{array}{l}\text { Sheep rabbit Immunoglobulin G (IgG)-Fluorescein } \\
\text { isothiocyanate (FITC) }\end{array}$ & $\begin{array}{l}\text { Purchased from Beijing Zhongshan Jinqiao Biotechnology } \\
\text { co., Ltd }\end{array}$ \\
\hline Restriction endonuclease (EcoRI) enzyme & From Takara \\
\hline Restriction enzyme Notl & From Takara \\
\hline T4 ligase & From Takara \\
\hline Taq polymerase & From Takara \\
\hline DNA Maker & From Takara \\
\hline Deoxynucleotide triphosphates (dNTPs) & Purchased from Beijing Tiangen Biochemical Co., Ltd \\
\hline Protein prestaining marker & Purchased from Beijing Tiangen Biochemical Co., Ltd \\
\hline Western blot color development kit & Purchased from Beijing Tiangen Biochemical Co., Ltd \\
\hline Membrane sealing fluid & Purchased from Beijing Tiangen Biochemical Co., Ltd \\
\hline Murine antihistidine his monoclonal antibody & Purchased from Beijing Tiangen Biochemical Co., Ltd \\
\hline Alkaline phosphatase labeled sheep anti-mouse IgG & Purchased from Beijing Tiangen Biochemical Co., Ltd \\
\hline
\end{tabular}


Nickel (NI) column

Agarose gel recovery kit

Small plasmid extraction kit

Low melting point agarose

Sodium dodecyl sulfate (SDS)

$30 \%$ acrylamide solution

Tris (hydroxymethyl) aminomethane hydrochloride (Tris- $\mathrm{HCl}$ $\mathrm{pH} 8.8$

Tris-HC1 $\mathrm{pH} 6.8$

WB color liquid

Restriction enzyme Sfi I

Anti-M13 antibody (Horseradish peroxidase (HRP))

Anti-E tag antibody (HRP)

Goat Anti-L1ama IgG (H\&L) (HRP)
General Electric (GE) corporation

Purchased from Beijing tiangen biochemical co., LTD

Purchased from Beijing Tiangen Biochemical Co., Ltd

Purchased from Shanghai Sangon Biological Engineering Co., Ltd

Purchased from Shanghai Sangon Biological Engineering Co., Ltd

Purchased from Shanghai Sangon Biological Engineering Co., Ltd

Purchased from Shanghai Sangon Biological Engineering Co., Ltd

Purchased from Shanghai Sangon Biological Engineering Co., Ltd

Purchased from Shanghai Sangon Biological Engineering Co., Ltd

Purchased from New England Biolabs ${ }^{\circledR}$ (NEB)

Purchased from Abcam

Purchased from Abcam

Purchased from Abcam

TABLE 2: MAIN INSTRUMENTS

\begin{tabular}{lc}
\hline Instruments & Source \\
\hline Inverted microscope & Nikon \\
Fluorescent microscope & Sikon \\
Digital camera & Henan Shuguang Jianshi Medical Equipment Group Co., Ltd. \\
Disposable syringe & Dyy-7c \\
Polyacrylamide gel electrophoresis apparatus & AKTAXROSS \\
Intelligent protein purification system & Thermo \\
Constant temperature table & Sanyo \\
Ultra-low temperature refrigerator & JY92- I \\
Cell ultrasonography & Norinco \\
Hq-60 vortex mixer & Tecan \\
Enzyme standard instrument & HP-9052 \\
Electric thermostatic nutrition box & ESCO \\
Super clean table & Hwy-100B \\
Constant temperature decolorizing table &
\end{tabular}

Methods:

Cytotoxicity test of MSTN nano antibody: Culture of myoblasts of immortalized mouse myoblast cell line $(\mathrm{C} 2 \mathrm{C} 12)$ is explained here. Cell resuscitation-Make preparation for protection and take out the $\mathrm{C} 2 \mathrm{C} 12$ cell line freezing tube from the liquid nitrogen tank. Quickly put it into a water bath at about $37^{\circ}$, shake it from time to time, try to melt it completely within $1 \mathrm{~min}$ and then take out the cells in the ultra-clean working table. After centrifugation at $1000 \mathrm{rpm}$ for 8-10 min, pour it out for the night, add appropriate Dulbecco's Modified Eagle Medium (DMEM) culture medium and inoculate it into the culture plate. The inoculation concentration is controlled at $1 \times 10 \% / 1$. Cells are cultured in incubator with $5 \%$ Carbon dioxide $\left(\mathrm{CO}_{2}\right)$ at $37^{\circ}$, the cell culture medium is changed within $24 \mathrm{~h}$ and then the culture is continued. When the cells covered the bottom of the plate, they are digested with $0.25 \%$ Ethylenediamine Tetraacetic Acid (EDTA) trypsin digestive solution and then cells are counted. $10^{3}$ $10^{4}$ cells are inoculated to 96 well cell culture plates and the grouping test is carried out when the cells grow to 90 $\%$ fusion.

Treatment of cells: The test is divided into four groups according to the different detection time. The concentration of the bivalent nano antibody Membrane Proteome Array (MPA) is 10, 20, 30 and $60 \mu \mathrm{g} / \mathrm{ml}$, respectively. One group 
is detected $12 \mathrm{~h}$ after treatment, the other group is detected 24 $\mathrm{h}$ after treatment. Each group is divided into four subgroups, which are three treatment subgroups and one control subgroup. Each treatment group is set with four replicates and each cell culture hole is set with one replicate. Using the inverted microscope ${ }^{[12,13]}$, the growth morphology of the cells is observed and recorded by image.

Detection of cytotoxicity by 3-(4,5-dimethylthiazol2-yl)-2,5-diphenyl tetrazolium bromide (MTT)-MTT cell technology is used to detect the proliferation and cytotoxicity of nano antibody to myoblasts.

According to the operation of cell culture as described above in this experiment, the number of cells is determined, the concentration of cell suspension is adjusted, $60 \mu \mathrm{l}$ cells (cell number 104/well) are inoculated into 96 well cell culture plate ${ }^{[14]}$ and cultured at $37^{\circ}$ with $5 \% \mathrm{CO}_{2}$ for $2 \mathrm{~h}$. When all cells adhere to the wall, the upper culture medium is carefully removed and diluted nano antibody is added. In the same way as the treatment given in the above experiment, the Phosphate Buffered Saline (PBS) control group, blank control group and the observation group with the concentration of $10,20,30,60 \mu \mathrm{g} / \mathrm{ml}$ of bivalent nano antibody MPA are set at the same time. Each group is set with zero adjustment holes (cell culture solution, MTT solution, formazan solution). Each group is set with four repeated average values and MTT is counted at $3 \mathrm{~h}, 6 \mathrm{~h}, 12 \mathrm{~h}, 24 \mathrm{~h}$ and $48 \mathrm{~h}$ respectively. The culture medium in each hole is discarded with a discharge gun, 20 $\mu \mathrm{l}$ fresh cell culture medium and $10 \mu \mathrm{l}$ MTT solution are added, and cultured continually for $4 \mathrm{~h}$. Then, the culture solution ${ }^{[15]}$ of each hole is discarded with a discharge gun and $30 \mu \mathrm{l} /$ hole formazan solution are added. The culture plate is placed on the shaking table and shaken for $10 \mathrm{~min}$ at a low speed. Then each hole becomes transparent purple solution, which represents the full dissolution of crystal. The absorbance value of each group is detected at Optical Density (OD) $490 \mathrm{~nm}$ of the microplate reader. All data are analyzed by two-way Analysis of Variance (ANOVA) with GraphPrism 6 software.

\section{Repeated verification of bivalent sheep MSTN nano antibody on mice:}

Animal experiment-30 Kunming mice of 1 mo old with a weight of $15 \mathrm{~g}$ are bought from Xinjiang Medical University and then the mice are fed in the laboratory for $3 \mathrm{~d}$ to observe the growth changes. The monitored Kunming mice are selected for subsequent experiments and randomly divided into three groups: PBS group, monovalent group, bivalent group, 10 in each group, for experimental verification. Injection and weighing experiments are carried out every other day. Each PBS group is injected with $30 \mu \mathrm{PBS}$ as the control group; the monovalent group is injected with $30 \mu \mathrm{l}(60 \mu \mathrm{g})$ monovalent sheep MSTN nano antibody; the bivalent group is injected with $30 \mu \mathrm{l}(60 \mu \mathrm{g})$ bivalent sheep MSTN nano antibody. The mice are weighted firstly, then injected and the state of mice are observed and recorded.

Anatomical observation of muscle volume changes: The mice are killed with their necks broken and their hind limbs are separated to weigh the two hind legs.

Making sections to observe the changes of muscle tissue: The adductor longus muscle of mouse leg is taken to make muscle fiber section and histological observation. The number of muscle fibers is statistically analyzed.

\section{RESULTS AND DISCUSSION}

Cell morphology is observed. The morphology of $\mathrm{C} 2 \mathrm{C} 12$ myoblasts does not change after being infected by MPA and Mitogen-Activated Protein Kinase (MPK). After 12 and $24 \mathrm{~h}$ of MPA treatment, compared with normal cells, there is no significant difference in the growth shape and quantity between PBS control cells and cells treated with different concentrations. The outline of the cells is clear, the cells are spindle or fibrous and the shape is long and thin (fig. 1 and fig. 2).

MTT test results are shown below. After two-factor ANOVA (interaction), the nano antibody of different concentrations has no toxic effect on $\mathrm{C} 2 \mathrm{C} 12$ cells. The cells grow regularly with the prolongation of culture time. At $12 \mathrm{~h}$, the number of cells reaches the peak. After 48 $\mathrm{h}$, the number of cells in each group decreases regularly. There is no significant difference between groups, $p>0.05$ and the experimental results are reliable (fig. 3).

30 Kunming mice are used in the experiment, from January 26, 2016 to February 22, 2016, for a total of $30 \mathrm{~d}$. Each PBS group is injected with $30 \mu \mathrm{PBS}$ as the control group; the monovalent group is injected with $30 \mu \mathrm{l}(60$ $\mu \mathrm{g})$ monovalent sheep MSTN nano antibody; the bivalent group is injected with $30 \mu \mathrm{l}(60 \mu \mathrm{g})$ bivalent sheep MSTN nano antibody.

After the experiment, the mice are treated. There is no difference in the final total body weight among the control group, monovalent nano antibody and bivalent nano antibody. There is no significant difference in promoting weight gain (Table 3 ).

The tissues between the two hind limbs are dissected and observed. It is found that the muscle of the bivalent sheep MSTN nano antibody group is fuller than that of the control group (fig. 4). The legs are separated and weighed separately (Table 4). 


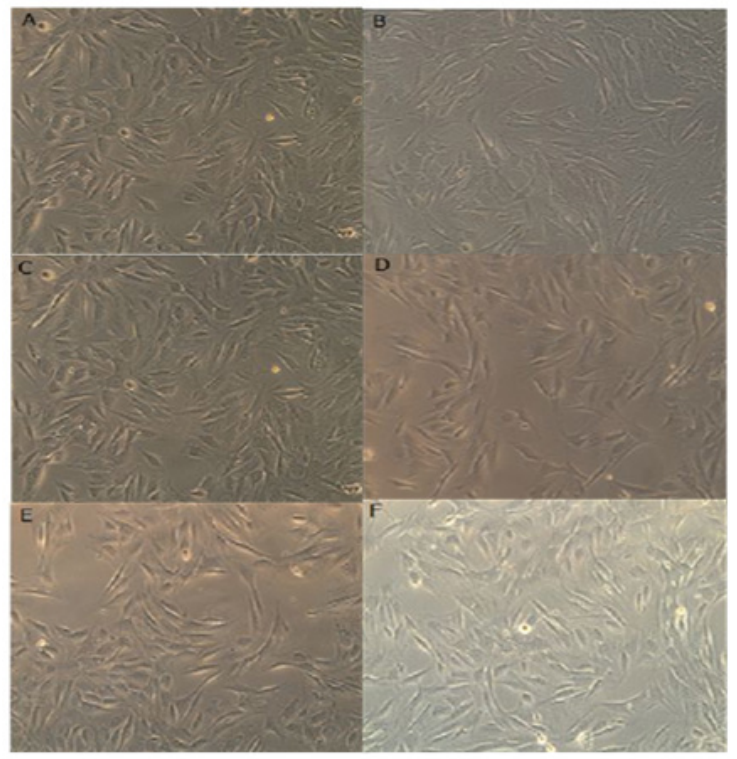

Fig. 1: Cell morphology after $12 \mathrm{~h}(20 \times)$, (A) Normal cells; (B) The role of the cells after PBS; (C-F) The role of the cells after polyclonal antibody protein for $10,20,30,60 \mu \mathrm{g} / \mathrm{ml}$, respectively

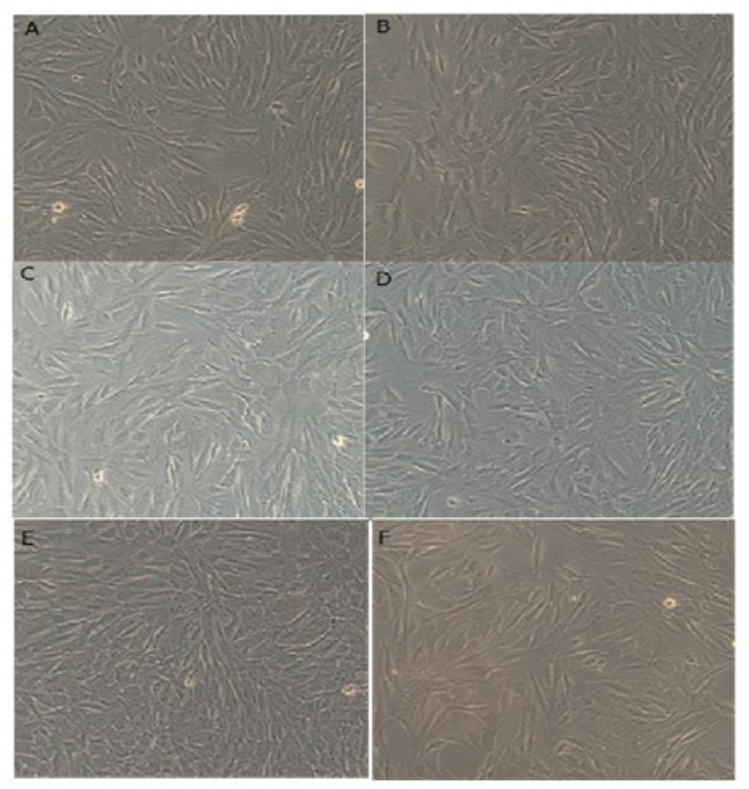

Fig. 2: Cell morphology after $24 \mathrm{~h}(200 \times)$, (A) Normal cells; (B) The role of the cells after PBS; (C-F) The role of the cells after polyclonal antibody protein for $10,20,30,60 \mu \mathrm{g} / \mathrm{ml}$, respectively
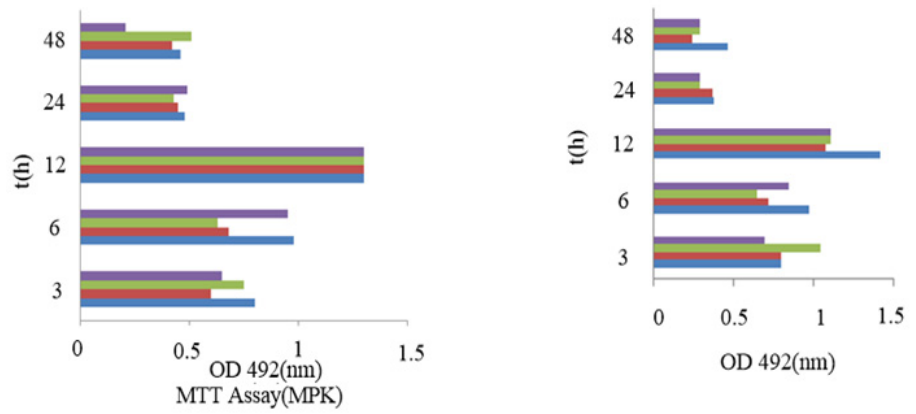

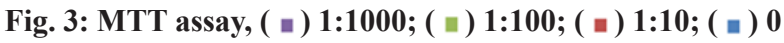


TABLE 3: MOUSE BODY WEIGHT

\begin{tabular}{ll}
\hline Group & Weight (g) \\
\hline PBS & $40.85 \pm 2.87$ \\
Monovalent & $40.78 \pm 2.17$ \\
Bivalent & $39.38 \pm 1.18$ \\
\hline
\end{tabular}

Note: PBS-PBS injected control group; Monovalent-Injection unit of sheep MSTN nanobodies; Bivalent-Sheep MSTN injection of bivalent nano antibody

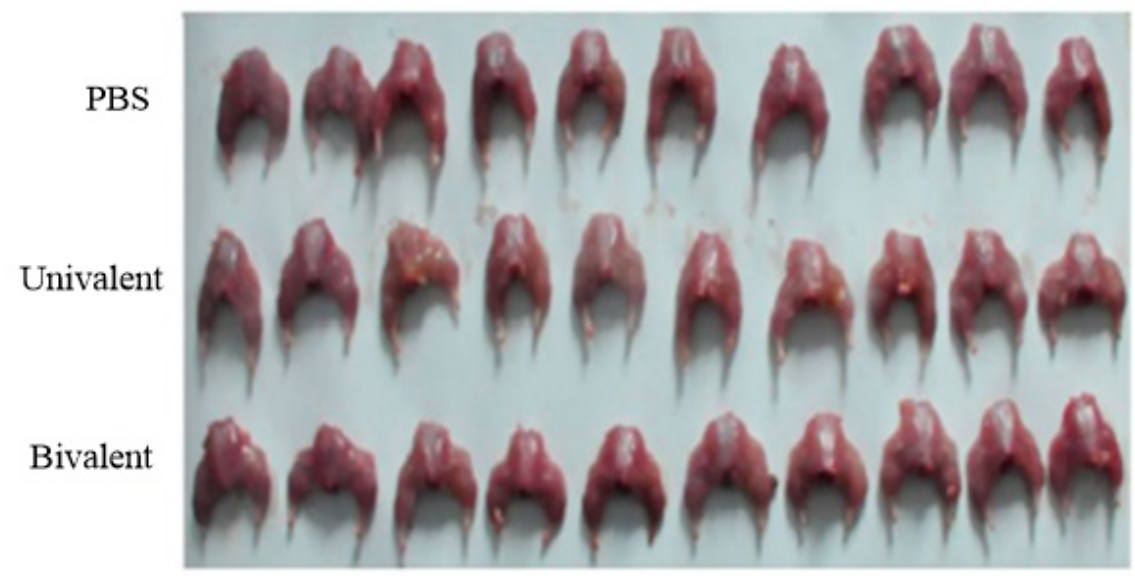

Fig. 4: Mouse two hind limbs muscle morphology diagram, PBS: PBS injected control group; Monovalent: Injection unit of sheep MSTN nanobodies; Bivalent: Sheep MSTN injection of bivalent nano antibody

TABLE 4: MOUSE HINDLIMB WEIGHT

\begin{tabular}{ll}
\hline Group & Weight (g) \\
\hline PBS & $5.33 \pm 0.33$ \\
Monovalent & $5.46 \pm 0.18$ \\
Bivalent & $5.62 \pm 0.31$ \\
\hline
\end{tabular}

Note: PBS-PBS injected control group; Monovalent-Injection unit of sheep MSTN nanobodies; Bivalent-Sheep MSTN injection of bivalent nano antibody

It can be observed that the bivalent nano antibody group has full muscle. The right hind legs are separated, compared and weighed, as shown in fig. 5.

It is found from leg weighing that the difference between bivalent nano antibody and monovalent nano antibody is very significant $(p=0.004<0.01)$ and the difference between bivalent nano antibody and control group is also very significant $(\mathrm{p}=0.000<0.01)$ (Table 5).

The two groups of pictures are enlarged and compared more intuitively. The hind limb of bivalent nano antibody group is significantly larger than that of monovalent nano antibody group and monovalent nano antibody group is larger than that of control group, as shown in fig. 6.

Because of the small size and easy to obtain, the experimental operation is simple and the effect is fast, so in this paper, the toxicity effect and muscle morphology of the sheep nano MSTN antibody in mice are verified. The whole mouse contains a large number of bones and other tissues. In the hind limb, the proportion of muscle is large and it is easy to separate, which is more representative. Therefore, the hind limb is separated and the muscle growth contrast experiment is carried out to determine the effect of sheep nano antibody.

The toxicity of MSTN nano antibody is not clear. Therefore, the proliferation and cytotoxicity of the nano antibody to myoblasts are detected by MTT technology. The safety of the antibody is determined by the toxicity grouping experiment at different concentrations. The experimental results show that (fig. 1 and fig. 2) there is no change in the morphology of the infected cells ${ }^{[16]}$. After 12 $\mathrm{h}$ and $24 \mathrm{~h}$ of treatment, the cells in the experimental pores treated with different concentrations have no significant difference in the growth shape and quantity. Therefore, it can be inferred that the nano antibodies of different concentrations have no toxicity to the cells. 


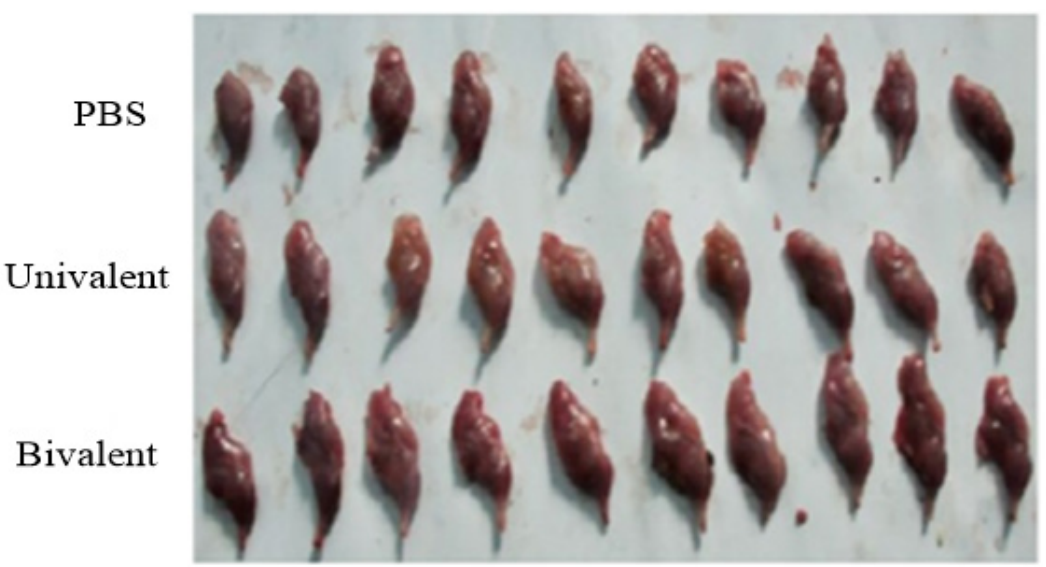

Fig. 5: Mouse right hind limb muscle morphology diagram, PBS: PBS injected control group; Monovalent: Injection unit of sheep MSTN nanobodies; Bivalent: Sheep MSTN injection of bivalent nano antibody

TABLE 5: MICE WITH UNILATERAL HINDLIMB WEIGHT

\begin{tabular}{ll}
\hline Group & Weight (g) \\
\hline PBS & $1.34 \pm 0.12$ \\
Monovalent & $1.59 \pm 0.13$ \\
Bivalent & $1.82 \pm 0.19$ \\
\hline
\end{tabular}

Note: PBS-PBS injected control group; Monovalent-Injection unit of sheep MSTN nanobodies; Bivalent-Sheep MSTN injection of bivalent nano antibody

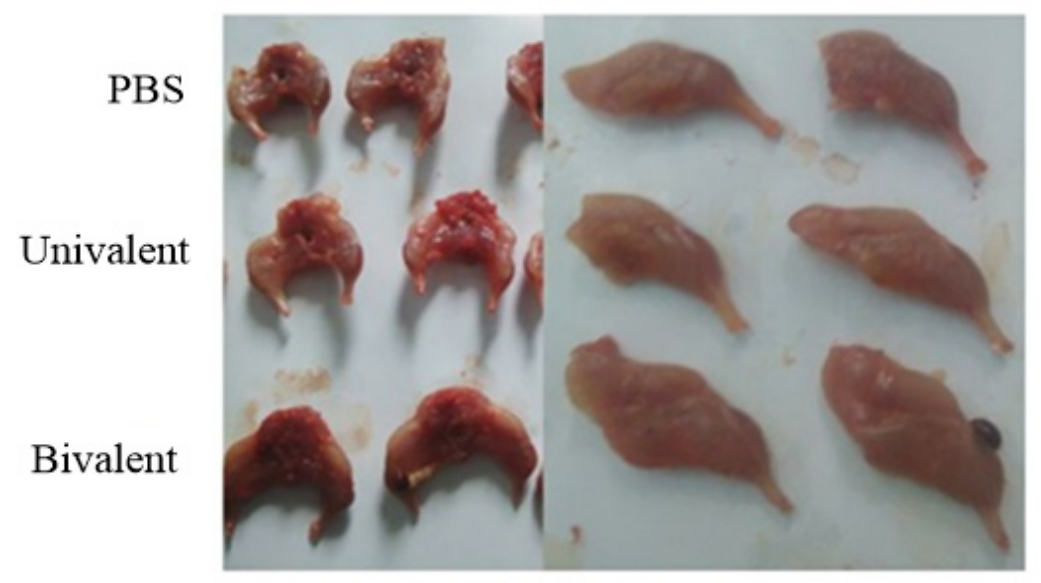

Fig. 6: Mouse two hind limbs and right hind limb muscle morphology diagram, PBS: PBS injected control group; Monovalent: Injection unit of sheep MSTN nanobodies; Bivalent: Sheep MSTN injection of bivalent nano antibody

Through the detection of cells at different time points, it is found that the number of cells increase regularly with the prolongation of culture time and reach the peak at $12 \mathrm{~h}$ (fig. 3), while the number of cells decrease regularly after $48 \mathrm{~h}$. The experimental results verify that MSTN nano antibody has no side effect on cell growth and muscle cells can grow normally after use.

Repeated experiments on mice verify that the nano antibody mainly has the effect of promoting muscle growth. By comparing the data of the two groups, it can be found that the bivalent nano antibody group has no significant difference with other groups in total body weight, but has significant difference with other groups in rear limb weight and is the heaviest group in each group. In Table 3, there is no significant difference between the bivalent nano antibody group and the other two groups, but in Table 5, the difference between the bivalent nano antibody group and the other two groups is extremely significant ${ }^{[17]}$. In the repeated experiments of mice, the bivalent nano antibody group is the lowest in total body weight, but the bivalent nano antibody group is the highest in leg muscle weight. It shows that the proportion of muscle in bivalent nano antibody group is the maximum and the muscle content in the body is also the maximum ${ }^{[18]}$. 
The muscle volume of the monovalent sheep MSTN nano antibody group is significantly smaller than that of the bivalent sheep MSTN nano antibody group, indicating that the effect of promoting muscle growth of the bivalent sheep MSTN nano antibody group is better than that of the monovalent sheep MSTN nano antibody group ${ }^{[19]}$. The body weight gain of monovalent sheep MSTN nano antibody group is similar to that of PBS control group and the net weight gain is similar to that of PBS control group, but the muscle volume is significantly larger than that of PBS control group; while that of bivalent sheep MSTN nano antibody group is almost smaller than that of PBS control group in terms of weight gain and net weight gain, but the muscle volume is much larger than that of PBS control group. Therefore, the main function of MSTN nano antibody is to promote muscle growth, especially the muscle growth of limbs.

The muscle tissue volume of the bivalent sheep MSTN nano antibody group is significantly larger than that of the monovalent sheep MSTN nano antibody group and there is also a significant difference between the monovalent sheep MSTN nano antibody group and the PBS control group. Through comparison, it is found that the growth promoting function of the bivalent sheep MSTN nano antibody group is significantly higher than that of the monovalent sheep MSTN nano antibody group ${ }^{[20,21]}$.

In this paper, the effect of sheep nano antibody on the movement of human tissue flap muscle is studied. By studying the toxic effect of sheep nano antibody on mouse C2C12 myoblasts and its effect on the morphology of tissue muscle, we can judge whether sheep nano antibody can be used in human tissue flap muscle, solve the problem of human muscle atrophy in medicine and recover and improve the ability of movement of human muscle. The cytotoxicity test of MSTN nano antibody shows that at different concentrations, the nano antibody would not affect the normal growth of cells over time, so it knows that the bivalent sheep MSTN nano antibody has no side effects on human tissue flap muscle cells and can be applied to human tissue flap muscle. Moreover, the bivalent sheep MSTN nano antibody has a greater impact on muscle morphology and the effect of bivalent sheep MSTN nano antibody on improving muscle morphology is particularly significant. In conclusion, sheep nano antibody is proved to be safe and reliable, which can be used to improve the movement ability of human tissue flap muscle and restore the muscle shape of patients with muscle atrophy.

\section{Conflict of interests:}

The authors declared no conflict of interest.

\section{REFERENCES}

1. Deng K, Ma T, Wang Z, TanTai W, Nie H, Guo Y, et al. Effects of Perilla frutescens seed supplemented to diet on fatty acid composition and lipogenic gene expression in muscle and liver of Hu lambs. Livest Sci 2018;211:21-9.

2. Çetin S, Yur F. Levels of trace elements in muscle and kidney tissues of sheep with fluorosis. Biol Trace Elem Res 2016;174(1):82-4.

3. Sen U, Sirin E, Yildiz S, Aksoy YÜ, Ulutas Z, Kuran M. The effect of maternal nutrition level during the periconception period on fetal muscle development and plasma hormone concentrations in sheep. Animal 2016;10(10):1689-96.

4. Yaghfoori S, Razmi GR, Mohri M, Razavizadeh AR, Movassaghi AR. An experimental ovine Theileriosis: The effect of Theileria lestoquardi infection on cardiovascular system in sheep. Acta Trop 2016;161:55-61.

5. Liu W, Chen X, Ni X. The modified temporalis muscle flap in reconstruction of palate and temporal deformity. Acta Otolaryngol 2017;137(8):899-902.

6. Rubayi S, Gabbay J, Kruger E, Ruhge K. The modified Girdlestone procedure with muscle flap for management of pressure ulcers and heterotopic ossification of the hip region in spinal injury patients: A 15-year review with long-term followup. Ann Plast Surg 2016;77(6):645-52.

7. Hwang K, Choi JH, Kim JH. Improvement of nasojugal groove and wrinkles following a skin-muscle flap elevation through a subciliary approach to the orbital rim. J Craniofac Surg 2016;27(3):741-4.

8. Dat AD, Loh IW, Bruscino-Raiola F. Free-flap salvage: muscle only versus skin paddle-an Australian experience. ANZ J Surg 2017;87(12):1040-3.

9. Singh M, Li H, Caterson EJ, Talbot SG. Innervated free gracilis muscle transfer: An experimental model. J Craniofac Surg 2016;27(6):1515-6.

10. Yano T, Okazaki M, Tanaka K, Tsunoda A, Aoyagi M, Kishimoto S. Feasibility and advantage of a musclesparing laterally based pericranial flap. J Craniofac Surg 2016;27(3):552-7.

11. Xiong L, Huang L, Shimo SP, Li W, Yang X, Yan P. Multiresidue method for the screening of benzimidazole and metabolite residues in the muscle and liver of sheep and cattle using HPLC/PDAD with DVB-NVP-SO $3 \mathrm{Na}$ for sample treatment. Chromatographia 2016;79(19):1373-80.

12. Yang HJ. Communication power supply monitoring system based on TCP/IP protocol. Chin J Power Sources 2016;40(6):1298-9.

13. Song HJ, Dong SW, Qu LL. Research on clock difference prediction using adaptive Kalman filter based on Sage window. Chin J Sci Instrum 2017;38:1809-16.

14. Peng W, Huang Q. Research on complex information system evolution process. J China Acad Electron Inf Technol. 2017;12(5):475-80.

15. Li XQ, Feng L, Zhao J. Design of the repetition-rate high voltage pulsed power by cold cathode trigger tube. J Power Supply 2016;14:33-7.

16. Sun HY. Based on multiple features fusion method of remote sensing image feature extraction. Comput Simul 2016;33(10):334-7.

17. Guo P, Sun Y, Li LH. Effect of Nano-TiO 2 on activity of Azotobacter in soil. J Jilin Univ (Sci Ed) 2017;55:1031-6. 
18. Ablikim B, Liu Y, Kerim A, Shen P, Abdurerim P, Zhou GH. Effects of breed, muscle type, and frozen storage on physicochemical characteristics of lamb meat and its relationship with tenderness. CyTA J Food 2016;14(1):109-16.

19. Takayama Y, Yokoo S, Makiguchi T, Komori T. Motor nerve preservation and muscle atrophy after pectoralis major musculocutaneous flap surgery for oromandibular reconstruction. J Craniofac Surg 2016;27(8):2055-60.

20. Bertheuil N, Cusumano C, Meal C, Harnoy Y, Watier E, Meunier B. Skin perforator flap pedicled by intercostal muscle for repair of a tracheobronchoesophageal fistula. Ann Thorac Surg 2017;103(6):e571-3.
21. Liu HP, Shao Y, Zhang D. Single-stage reconstruction of eyebrow defect using a VY advancement pedicle flap based on the orbicularis oculi muscle. J Craniofac Surg 2017;28(6):e521-2.

This is an open access article distributed under the terms of the Creative Commons Attribution-NonCommercial-ShareAlike 3.0 License, which allows others to remix, tweak, and build upon the work non-commercially, as long as the author is credited and the new creations are licensed under the identical terms

This article was originally published in a special issue, "Diagnostic and Therapeutic Advances in Biomedical Research and Pharmaceutical Sciences"

Indian J Pharm Sci 2021:83(5)Spl Issue “259-267” 